\title{
EXPERIENTIAL LEARNING IN LEADERSHIP DEVELOPMENT: SELECT PROGRAM AT MCMASTER UNIVERSITY
}

\author{
Minha R. Ha, Faculty of Engineering, McMaster University | englead@mcmaster.ca
}

\section{INTRODUCTION}

This presentation provides a detailed overview of SELECT in its two pilot years, the learning from which informs the model of student leadership, learning and development that guides ongoing program decisions. For SELECT, the new co-curricular leadership development program open to undergraduate engineering students at McMaster University, student ownership has been the key driver of program development from its inception to ongoing implementation. The efforts to align the program content and processes to the program vision and principles are met with a unique opportunity and challenge of strengthening and sustaining student ownership (and authorship) through cohorts, and learning as educators to understand and design for true experiential pedagogy in given context.

\section{PROGRAM DESIGN}

As part of the experiential learning thrust in the Faculty of Engineering, a common vision for SELECT continues to be an integrated student community and empowered leadership capabilities of teams and individual students. Two co-curricular Tiers were created - each with an application, initial consultation, orientation, four workshop modules and feedback mechanisms - and complemented by optional Ambassador and Mentorship programs.

Tier 1 modules included individual and group exercises, facilitated by alumni and colleagues from the industry. Group activities often incorporated game principles (i.e. competition, time constraint, concrete tasks, flexible routes) and active communication among members, after which facilitators posed questions for feedback, reflecting on the team dynamics and behavior. The facilitators were then asked more specific questions relating to real life situations.

Tier 2 focused on bringing experiences and insights of facilitators and students as the main content of the modules, while pre-designed packages provided standalone resources for student leaders. Facilitator presentations were followed by exercises and large group discussions, after which the facilitators joined the small group discussions to delve deeper into real issues. All Tier 2 members were required to be serving active roles in their student organisations. Each group then presented their take-home principles and action plans.
Ambassadors were individuals who committed to improving student experience and developing their skills through making a direct contribution to SELECT implementation. In pilot year 1 , the Ambassadors conducted independent research and presented to the group. In year 2, the Ambassadors hosted roundtable and special training sessions with local professionals, and served on faculty committees or special projects. In both years they were responsible for organising SELECT events and making input to ongoing program development.

\section{DISCUSSION}

Analysis of the results from employed program approaches posed useful implications for resource allocation, conceptualising the leaners and the learning process, and program evaluation. For example, creating early contact between staff and students proved effective for engagement and empowerment. Personal relationships built among peers and with staff were critical in communication, quick mobilisation and creativity. In facilitating student ownership and initiative, students responded most strongly to well-communicated vision, thought-provoking questions, and concrete deliverables. The relationships could not uniform, however. Supporting the 'co-labourer' role of student leaders with resource effectiveness poses an interesting challenge to the operational model of SELECT.

Students are viewed as not only important stakeholders and resources to the theory and practice of leadership development, but also co-authors of such knowledge. Inquiring students about their leadership development process highlighted students' ability to reframe posed questions, provide unique perspectives while making connections and comparisons to others', challenge assumptions and function well in both divergent and convergent deliberations. Ongoing dialogue and collaboration with students are expected to be critical for well-situated design of program evaluation and measurement of outcomes.

In sum, both the anticipated and unexpected results of SELECT will likely depend on the quality of stakeholder relationships and changes fostered within and in relation to SELECT. One key to effectuating the transformative potential of student, faculty and community engagement may be found in addressing the challenge of building continuity across cohorts. 